\title{
Stromal vascular fraction injection to treat intractable radiation-induced rectovaginal fistula
}

\author{
Mijung Kim, Dae Hyun Lew, Tai Suk Roh, Seung Yong Song \\ Department of Plastic and Reconstructive Surgery, Yonsei University College of Medicine, Seoul, Korea
}

\begin{abstract}
Rectovaginal fistula, which can arise after an injury to the vaginal canal or rectum, is a troublesome obstacle for patients' everyday life. In most cases, it can be covered with a local flap, but previous radiation therapy increases the recurrence rate, making it especially difficult to cure. As the application of stromal vascular fraction (SVF) obtained from enzymatically digested autologous adipose tissue has become increasingly common, several reports have advocated its effectiveness for the treatment of refractory wounds. In light of the angiogenic, regenerative characteristics of SVF, it was incorporated as a treatment option in two cases of rectovaginal fistula discussed here. As described in this report, irradiated rectovaginal fistulas in rectal cancer patients were successfully treated with SVF injection, and we suggest SVF as a feasible treatment option for cases of rectovaginal fistula that would otherwise be very difficult to cure.
\end{abstract}

Keywords Rectovaginal fistula / Fat graft / Mesenchymal stem cell / Radiation effect

\author{
Correspondence: Seung Yong Song \\ Department of Plastic and \\ Reconstructive Surgery and Institute \\ for Human Tissue Restoration, Yonsei \\ University College of Medicine, 50-1 \\ Yonsei-ro, Seodaemun-gu, Seoul \\ 03722 , Korea \\ Tel: +82-2-2228-2214 \\ Fax: +82-2-393-6947 \\ E-mail: iceberg554@naver.com
}

\section{INTRODUCTION}

Rectovaginal fistula (RVF) is physically and psychosocially stressful for patients and remains a challenging condition for surgeons due to its high risk of recurrence. The treatment options for RVF are as various as its causes, which include obstetric trauma, radiation, malignancy, inflammatory disorders such as Crohn disease, or iatrogenic injury from a surgical procedure $[1,2]$. Diverting ostomy, a transrectal or transvaginal advancement flap, perineoplasty, fibrin glue, fistula plug, fistulectomy with Seton placement, gracilis muscle transposition, or an autologous fat graft are some options to consider for the treatment of RVF, but successful closure of the defect is not guaranteed, especially when the fistula is a result of radiation or a previous pelvic operation $[3,4]$.

The adipose stromal vascular fraction (SVF) can be obtained by enzymatic digestion of lipoaspirate or by mechanically processing adipose tissue, and contains a heterogeneous pool of cells including pericytes, mesenchymal stem cells, endothelial cells, and macrophages. With expectations for the progenitor activities of SVF and adipose tissue-derived stem cells, they have already been tried as a treatment for perianal fistula in Crohn disease $[5,6]$ or complex cryptoglandular fistula-in-ano [7]. Recent studies exploring the effectiveness of SVF for perianal fistula tended to focus on cases involving Crohn disease, and to the authors' knowledge, studies specifically exploring SVF application in the setting of radiation- and surgery-induced fistula are lacking. RVF in irradiated patients should be considered a different entity from RVF in other clinical settings, due to its unique pathophysiology and the characteristics of the surrounding tissue affected by radiation. Hence, in this report of two cases of radiated RVF, we hope to provide insights into the implications 
that SVF has as a feasible treatment option in this challenging clinical setting.

\section{CASES}

This study was approved by the Internal Review Board of Yonsei University Severance Hospital (IRB No. 4-2020-0806). Patients were informed of the risks and benefits of all the procedures and informed consent was obtained.

\section{Case 1}

A 61-year-old woman who was diagnosed with rectal cancer underwent concurrent chemoradiotherapy (radiation dose of $5,000 \mathrm{cGy}$ ), followed by robot-assisted low anterior resection. She had an ileostomy repair 5 months later, but ileostomy formation was repeated due to an RVF. The gynecologic surgery team attempted fistula repair, but symptoms developed again after she had an ileostomy repair. The patient was then referred to the current reconstructive team. A vaginal wall defect was noted in the 7-o'clock direction when the patient was placed in the lithotomy position. RVF repair was planned by coverage with a local flap and SVF injection. A total of $100 \mathrm{~mL}$ of fat was manually aspirated from the lower abdomen and transferred into a Smart X kit (Dongkoo Bio \& Pharma Co., Seoul, Korea), which then underwent enzymatic digestion with collagenase 1 , shaking and incubation, centrifugation at $1,000 \times g$ for 2 minutes, enzymatic washing three times, and filtration. As a result, $3 \mathrm{~mL}$ of SVF was obtained. After debridement and transposition of a vaginal mucosal Limberg flap, the SVF was injected to the defective posterior vaginal wall (Fig. 1). During the operation, loop ileostomy was done by the colorectal surgery team. Rectoscopy was carried out 5 months after surgery, and no air leakage or discharge from the vagina was noted. The patient underwent ileostomy repair, and did not show any signs of recurrence at the last visit, 1 year after surgery.

\section{Case 2}

A 59-year-old woman underwent preoperative chemotherapy and a short course of radiotherapy of 2,500 cGy due to rectal cancer. She received a laparoscopic low anterior resection and total laparoscopic hysterectomy with bilateral salpingo-oophorectomy. Three months later, she complained of fecal discharge from the vagina and an unsuccessful attempt to perform primary repair was made by the colorectal surgery team. She was referred to the plastic surgery department. A posterior vaginal mucosal defect in the 6-o'clock direction was noted (Fig. 2). In the first operation, $150 \mathrm{~mL}$ of abdominal fat was harvested, of which $120 \mathrm{~mL}$ was used to extract $5 \mathrm{~mL}$ of SVF. Along with the

\section{Fig. 1. Intraoperative photo of case 1}

(A) Preoperatively, a rectovaginal fistula was noted on the posterior vaginal wall. (B) After coverage with a local flap, a stromal vascular fraction injection was performed.
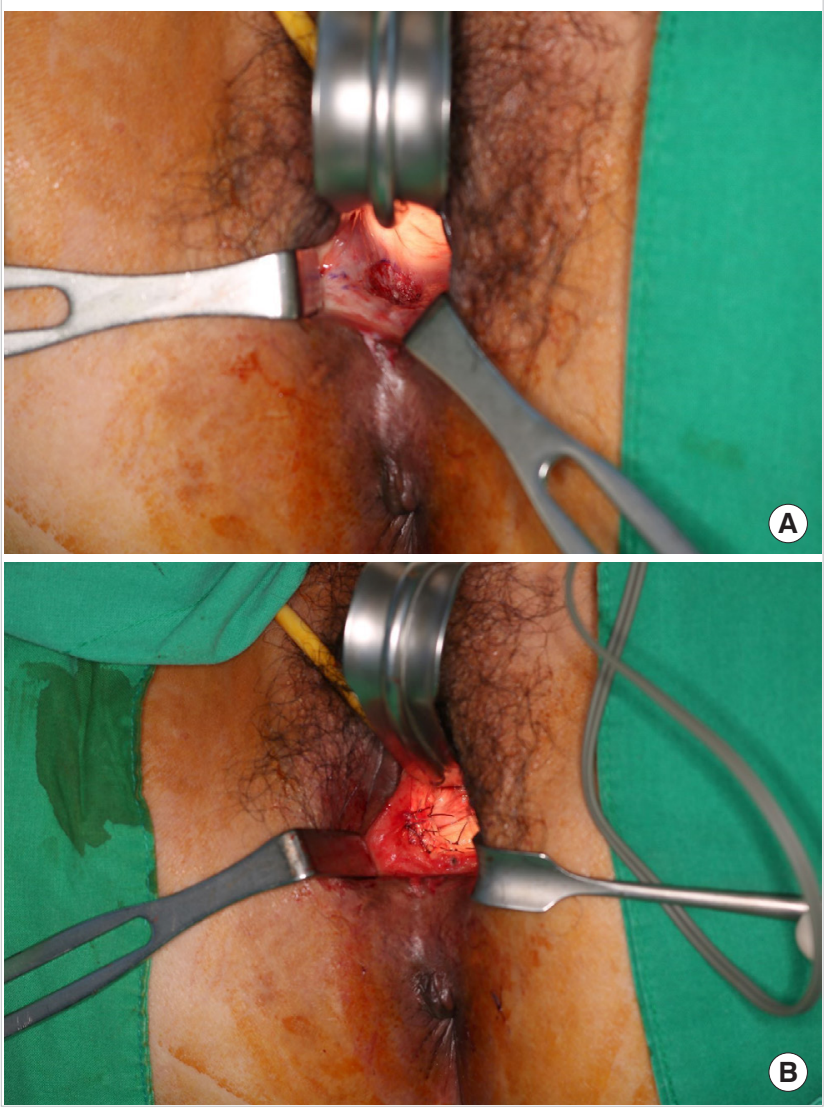

Fig. 2. Intraoperative photo of case 2

A rectovaginal fistula (black arrow) was noted at the time of the second cell-assisted lipotransfer.

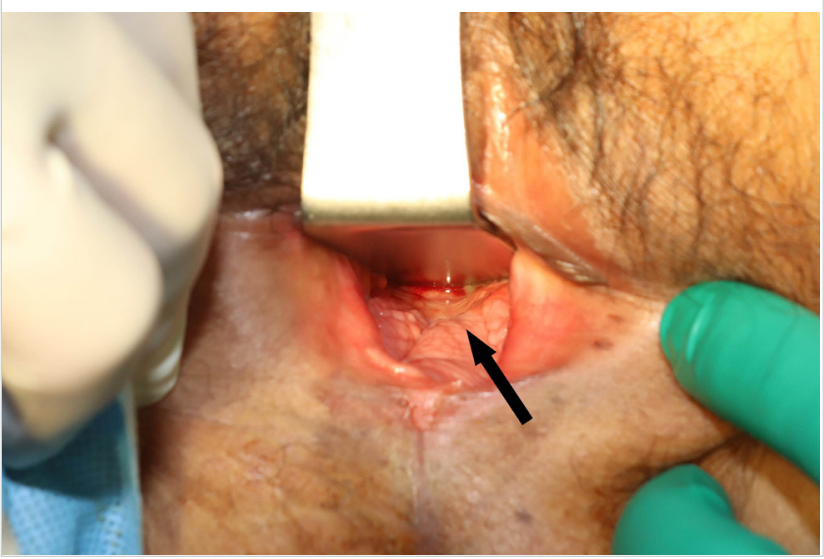

SVF, a 30-mL fat graft was placed at the defect. After 1 month, she reported a notably reduced, but still extant, fecal discharge from the vagina, and the second operation was planned 3 months later. Again, $150 \mathrm{~mL}$ of fat was aspirated from the medi- 
al thigh and $5 \mathrm{~mL}$ of SVF was obtained. Adding $23 \mathrm{~mL}$ of centrifuged fat, a total of $28 \mathrm{~mL}$ of cell-assisted lipotransfer was done to the RVF. The patient did not show any clinical sign of recurrence when she visited the clinic 5 months later, and there was no evidence of a remnant fistula on a postoperative magnetic resonance imaging scan.

\section{DISCUSSION}

Although the exact mechanism behind the therapeutic effect of SVF has yet to be clarified, we can speculate that certain characteristics of SVF may exert beneficial actions in the setting of RVF occurring in a previously irradiated tissue. SVF is thought to be pro-angiogenic in that it provides a niche for angiogenesis by providing cell phenotypes associated with blood vessels, such as endothelial progenitor cells and adipose tissue-derived mesenchymal stem cells [8]. Radiation causes alterations in blood flow, resulting in ischemia around the target tissue, which then leads to desquamation, erythema, edema, fibrosis, and necrosis [9]. Rigotti et al. [10] observed neovessel formation and improved hydration in radiation-damaged tissue treated with purified autologous lipoaspirate.

Case 1 in our report developed an RVF following radiotherapy and rectal cancer surgery and underwent unsuccessful fistula repair surgery before she was referred to the current reconstruction surgery team. Since the tissue involving the fistula was expected to be poorly vascularized and fibrotic due to previous operations and radiation injury, we tried to assist the healing process by injecting SVF. Hasdemir et al. [11] experimentally showed that adipose-derived stem cells improved skin flap survival and a similar approach was undertaken for this case. Considering that no bulky lipotransfer was carried out in this caseinstead, only a thin mucosal flap was used-we expect that the angiogenic and regenerative effects of SVF could have assisted in the healing of the friable tissue.

Encouraged by the excellent results observed in case 1, the RVF of case 2 was treated with two serial microfat grafts accompanied by $5 \mathrm{~mL}$ of SVF, without performing more invasive surgical repair. Taking into consideration that the previously irradiated tissue surrounding the fistula must have impeded the survival of the grafted fat, the authors conclude that SVF played a regenerative role in the successful closure of the fistula with an autologous fat graft, avoiding more invasive surgery, which would have caused further damage to the surrounding tissue.

The pathogenesis of radiotherapy-induced tissue injuries involves multiple factors. The rapidly dividing cells of the mucosal epithelium and endothelium are radiosensitive, as well as the stem cells responsible for the maintenance of the mucosal barri- er, meaning that radiotherapy has a long-lasting effect on the regenerative capacity of irradiated tissue [12]. The therapeutic approach employed in this report was rooted in the hypothesis that by repopulating the irradiated tissue with the adipose-derived mesenchymal stem cells in SVF, the stem cell-depleted tissue may regain regenerative capacity for closure of the RVF. Indeed, despite previous failures of surgical correction, we observed a very promising effect of SVF as a treatment for refractory RVF. In the authors' method, SVF was injected as it was extracted via the Smart X system intraoperatively, without further isolation or expansion of stem cells. Although large, controlled studies directly comparing SVF and isolated adipose-derived stem cells are scarce, a few studies have suggested that SVF may be clinically advantageous over adipose tissue-derived stem cells per se, considering its quicker isolation and heterogeneity [1315]. Especially in light of the consequences of radiation history, the multipotent and hematopoietic cell population in SVF is expected to achieve an optimal regenerative milieu for the cure of damaged tissue.

This is a report of the authors' experience of utilizing SVF to treat RVF patients with a history of radiation treatment. Larger case series and further studies are expected to clarify actual effectiveness and long-term effects of SVF for the treatment of RVF. Additionally, the therapeutic protocol should be refined in the future to include an optimal dose, indications for accompanying surgical interventions, and a clearly established isolation and injection process.

\section{NOTES}

\section{Conflict of interest}

SYS, a deputy editor of Archives of Plastic Surgery, is the corresponding author of this article. However, he played no role whatsoever in the editorial evaluation of this article or the decision to publish it. Except for that, no potential conflict of interest relevant to this article was reported.

\section{Ethical approval}

The study was approved by the Institutional Review Board of Yonsei University Severance Hospital (IRB No. 4-2020-0806) and performed in accordance with the principles of the Declaration of Helsinki. Written informed consent was obtained.

\section{Patient consent}

The patients provided written informed consent for the publication and the use of their images. 


\section{Author contribution}

Conceptualization: SY Song. Methodology: SY Song. Project administration: DH Lew, TS Roh, SY Song. Writing-original draft: M Kim. Writing-review \& editing: M Kim, SY Song.

\section{ORCID}

Mijung Kim

Dae Hyun Lew

Tai Suk Roh

Seung Yong Song https://orcid.org/0000-0002-7126-6661 https://orcid.org/0000-0002-2625-5664 https://orcid.org/0000-0001-8681-159X https://orcid.org/0000-0002-3145-7463

\section{REFERENCES}

1. Champagne BJ, McGee MF. Rectovaginal fistula. Surg Clin North Am 2010;90:69-82.

2. Corte H, Maggiori L, Treton X, et al. Rectovaginal fistula: what is the optimal strategy? An analysis of 79 patients undergoing 286 procedures. Ann Surg 2015;262:855-60.

3. Ryoo SB, Oh HK, Ha HK, et al. Outcomes of surgical treatments for rectovaginal fistula and prognostic factors for successful closure: a single-center tertiary hospital experiences. Ann Surg Treat Res 2019;97:149-56.

4. Norderval S, Lundby L, Hougaard H, et al. Efficacy of autologous fat graft injection in the treatment of anovaginal fistulas. Tech Coloproctol 2018;22:45-51.

5. Garcia-Olmo D, Herreros D, Pascual M, et al. Treatment of enterocutaneous fistula in Crohn's disease with adipose-derived stem cells: a comparison of protocols with and without cell expansion. Int J Colorectal Dis 2009;24:27-30.

6. Laureti S, Gionchetti P, Cappelli A, et al. Refractory complex Crohn's perianal fistulas: a role for autologous microfragmented adipose tissue injection. Inflamm Bowel Dis 2020; $26: 321-30$
7. Borowski DW, Gill TS, Agarwal AK, et al. Autologous adipose-tissue derived regenerative cells for the treatment of complex cryptoglandular fistula-in-ano: a report of three cases. BMJ Case Rep 2012;2012:bcr2012006988.

8. Andia I, Maffulli N, Burgos-Alonso N. Stromal vascular fraction technologies and clinical applications. Expert Opin Biol Ther 2019; 19:1289-305.

9. Nguyen A, Guo J, Banyard DA, et al. Stromal vascular fraction: a regenerative reality? Part 1: current concepts and review of the literature. J Plast Reconstr Aesthet Surg 2016; 69:170-9.

10. Rigotti G, Marchi A, Galie M, et al. Clinical treatment of radiotherapy tissue damage by lipoaspirate transplant: a healing process mediated by adipose-derived adult stem cells. Plast Reconstr Surg 2007;119:1409-22.

11. Hasdemir M, Agir H, Eren GG, et al. Adipose-derived stem cells improve survival of random pattern cutaneous flaps in radiation damaged skin. J Craniofac Surg 2015;26:1450-5.

12. Chang PY, Qu YQ, Wang J, et al. The potential of mesenchymal stem cells in the management of radiation enteropathy. Cell Death Dis 2015;6:e1840.

13. Bora P, Majumdar AS. Adipose tissue-derived stromal vascular fraction in regenerative medicine: a brief review on biology and translation. Stem Cell Res Ther 2017;8:145.

14. Serrero M, Grimaud F, Philandrianos C, et al. Long-term safety and efficacy of local microinjection combining autologous microfat and adipose-derived stromal vascular fraction for the treatment of refractory perianal fistula in Crohn's disease. Gastroenterology 2019;156:2335-7.

15. Guo J, Nguyen A, Banyard DA, et al. Stromal vascular fraction: a regenerative reality? Part 2: mechanisms of regenerative action. J Plast Reconstr Aesthet Surg 2016;69:180-8. 